\title{
Visibility Representations of Complete Graphs
}

\author{
Robert Babilon, Helena Nyklová^, Ondřej Pangrác, and Jan Vondrák \\ Department of Applied Mathematics \\ Charles University \\ Malostranské nám. 25, Prague, Czech Republic \\ \{babilon, nyklova, pangrac, vondrak\}@kam.ms.mff.cuni.cz
}

\begin{abstract}
In this paper we study 3-dimensional visibility representations of complete graphs. The vertices are represented by equal regular polygons lying in planes parallel to the $x y$-plane. Two vertices are adjacent if and only if the two corresponding polygons see each other i.e. it is possible to construct an abscissa perpendicular to the $x y$-plane connecting the two polygons and avoiding all the others.

We give the bounds for the maximal size $f(k)$ of a clique represented by regular $k$-gons: $\left\lfloor\frac{k+1}{2}\right\rfloor+2 \leq f(k) \leq 2^{2^{k}}$ and we present a particular result for triangles: $f(3) \geq 14$.
\end{abstract}

\section{Introduction}

Consider a finite number of equal regular $k$-gons located in parallel planes so that their corresponding edges are parallel (we can only shift each polygon in its plane). We say that two polygons can see each other if there is a line segment perpendicular to the planes, which connects the two polygons and does not intersect any other. Let $f(k)$ denote the maximum number of $k$-gons which can be placed so that each pair of them can see each other.

Recently the result is known for squares (maximum clique represented by squares is $K_{7}$ ) and for discs (a clique of arbitrary size can be represented by discs). Both these result were shown by Fekete, Houle and Whitesides in [FHW].

We can assume that the planes are perpendicular to the $z$-axis, and we consider the projection of the polygons into the $x y$-plane. Clearly, only the $z$-order of the polygons is important, not the exact $z$-coordinates. We number the polygons according to the ordering on $z$-coordinate. We encode the position of each polygon by $k$ coordinates (which are not independent); each of them is measured in the direction perpendicular to one of the edges. (For example, we have 3 coordinates for a triangle, but only two of them are independent.) The basic idea is that the ordering of the polygons in these coordinates captures the geometric properties of our configuration completely. Note that although the exact values of two coordinates determine the location of the triangle uniquely, the ordering in two coordinates is not sufficient to describe the configuration. In Figure 1, the ordering in coordinates $\pi$ and $\rho$ is the same, but the $\sigma$-ordering of the triangles differ and the two configurations are not equivalent.

* Research supported by grant GAUK 159/99 


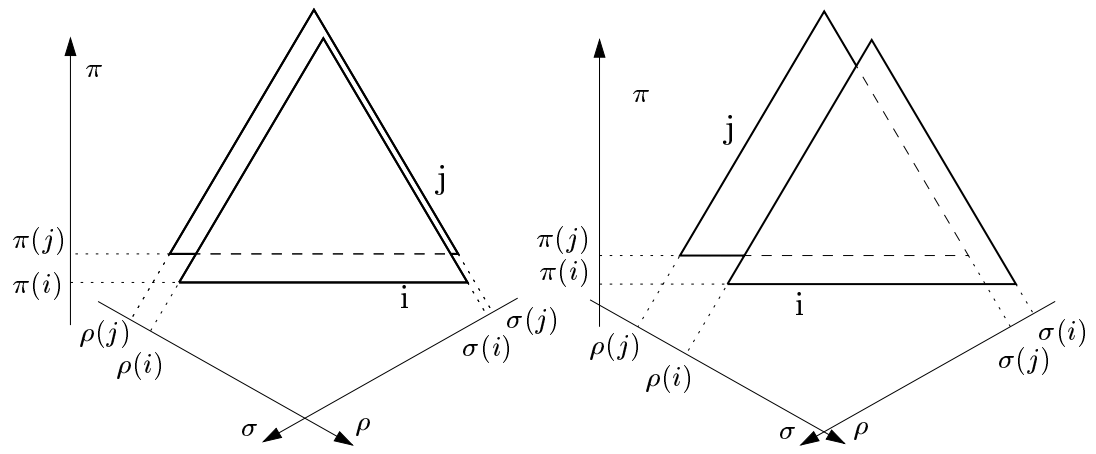

Fig. 1.

Without loss of generality we can assume that no two coordinates are equal, thus for any pair $i \neq j$ of $k$-gons we have $\pi(i) \neq \pi(j)$ on any axes.

From now on we use the expression configuration for a system of $k$-gons such that every $k$-gon sees all the others.

\section{General properties}

Lemma 1 If we allow rotation (i.e. we do not insist on parallelism of the corresponding edges) we can construct the arbitrarily large configuration of half-planes (and $k$-gons for arbitrary $k$ ).

Proof. Consider a point $A$ and a half-plane $\rho$ such that $A$ lies in the interior of $\rho$. First construct a half-plane $\rho_{1}$. Rotate it by the angle $\alpha_{1}=45^{\circ}$ with the center in $A$ and we obtain half-plane $\rho_{2}$. Every other half-plane we obtain as a rotated copy of the previously constructed half-plane, where $\alpha_{i}=\alpha_{i-1} / 2$. And we have a configuration of arbitrary number of half-planes. See Figure 2. The bounding line of $\rho_{i}$ is crossing the boundaries of $\rho_{1}, \rho_{2}, \ldots, \rho_{i-1}$ and therefore $\rho_{i}$ sees $\rho_{j}, j<i$.

Claim 2 For every natural $k, f(k) \geq\left\lfloor\frac{k+1}{2}\right\rfloor+2$.

Proof. Consider the system of $\left\lfloor\frac{k+1}{2}\right\rfloor$ half-planes seeing each other such that their angles with the horizontal $x$-coordinate are $0, \frac{2 \pi}{k}, 2 \frac{2 \pi}{k}, 3 \frac{2 \pi}{k}, \ldots,\left\lfloor\frac{k-1}{2}\right\rfloor \frac{2 \pi}{k}$. This can be done by taking the horizontal line $p_{1}$ with an orientation from the left to the right side and choosing a point $A$ not lying on $p_{1}$ as a center point of the rotation. Every next line $p_{i+1}$ arises from the previous $p_{i}$ through the counterclockwise rotation by the angle $\frac{2 \pi}{k}$ and shifting by $\delta$ along the $z$-coordinate. The half-planes are determined by the lines $p_{i}$ and the point $A$ which lies on them.

Now take sufficiently large $k$-gons $P_{i}$ (so that every $P_{i}$ contains all the intersection points of $p_{i}$ and all the other $p_{j}$ ) and align them to the boundaries of the corresponding half-planes. In such a way we obtain $\left\lfloor\frac{k+1}{2}\right\rfloor k$-gons seeing each other. 


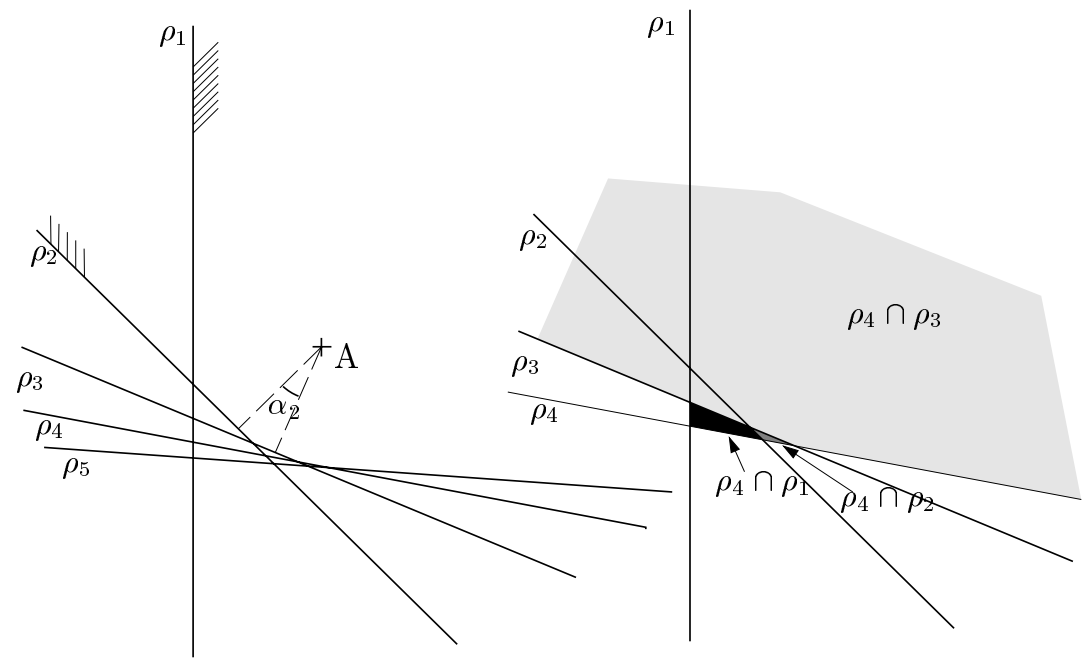

Fig. 2. Half-planes, if we allow rotation.

Moreover, we can add two more $k$-gons, one to the top and the second to the bottom of the configuration, centers of them lying not far from the intersection point of the first $\left(p_{1}\right)$ and the last $\left(p_{\left\lfloor\frac{k+1}{2}\right\rfloor}\right)$ line. These new $k$-gons see each other (because the intersection of complements of the first and the last half-plane is not empty) and also see all the reminding $k$-gons.

There are together (for each $k$ ) at least $\left\lfloor\frac{k+1}{2}\right\rfloor+2 k$-gons seeing each other.

The example for the case $k=6$ is shown in Figure 3 . We have three halfplanes (lines), three hexagons adjacent to the respective lines (2, 3 and 4), one hexagon on the top (1) and one hexagon on the bottom (5). There are together $\left\lfloor\frac{6+1}{2}\right\rfloor+2=5$ hexagons.

\section{Corollary 3}

$$
\lim _{k \rightarrow \infty} f(k)=\infty
$$

Proof. Immediately follows from the lower bound of $f(k)$.

Furthermore if we take the limit as $k \rightarrow \infty$ the $k$-gon approaches the shape of a disc. For discs we can use the same construction as we used in the proof of Lemma 1. The boundary lines of the half-planes are the touching lines of the corresponding discs and $\left|\rho_{i}, A\right|<r$. We have the same result as shown in [FHW] - every complete graph can be represented by discs.

Claim 4 For every natural $k, f(k) \leq 2^{2^{k}}$.

Lemma 5 If there is a sequence of three polygons which is monotone in each ordering, then there are two polygons such that one of them does not see the other one. 


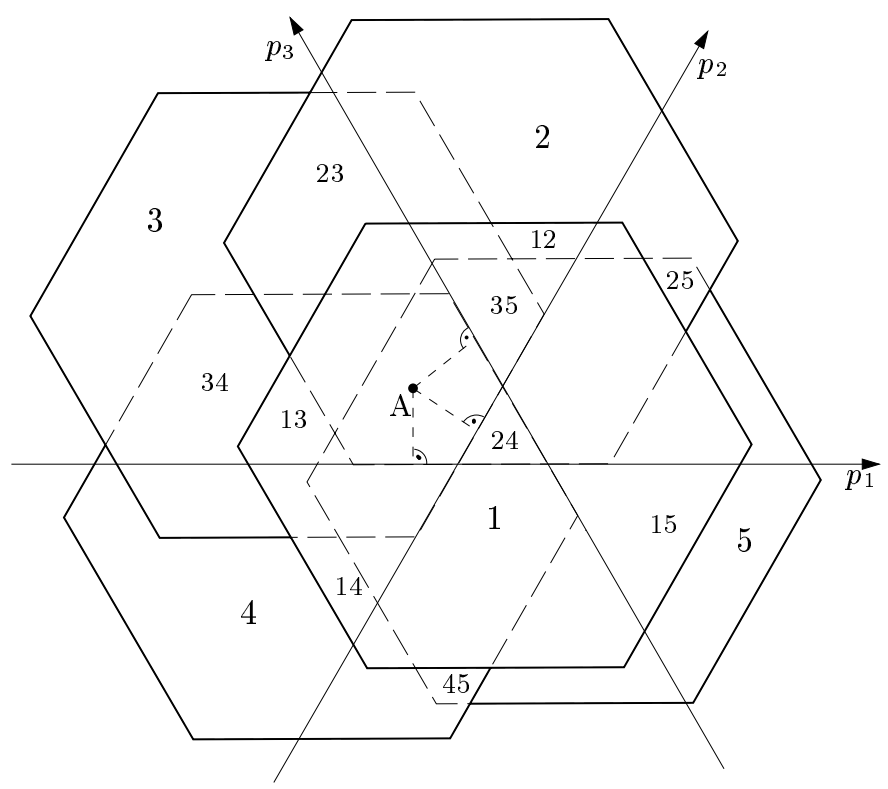

Fig. 3. $f(6) \geq 5$

Proof. If we have a sequence of three polygons monotone in each ordering, the middle one (in $z$-order) is the middle one in the ordering on each coordinate. Hence the common area of the first and the third one is contained in the second one. So they cannot see each other. (See Fig. 4.)

Proof of Claim 4. We use the Erdős-Szekeres theorem, which says that any sequence of $(a-1)(a-1)+1$ distinct numbers contains a monotone subsequence of the length $a$. We have a sequence $\left\{\left(x_{1}, x_{2}, \ldots, x_{k}\right)_{i}\right\}_{i=1}^{n} \in \mathbb{N}^{k}$ and we want to find a subsequence of the length 3 monotone in each coordinate. We proceed by induction on $k$.

Let us have a sequence $\left\{x_{i}\right\}_{i=1}^{n}, x_{i} \in \mathbb{N}$. Hence by Erdős-Szekeres theorem if $n_{1}=(3-1)(3-1)+1=5=2^{2^{1}}+1$ for $k=1$ we can find a monotone subsequence of the length 3 . For $n_{k}=\left(n_{k-1}-1\right)\left(n_{k-1}-1\right)+1=\left(2^{2^{k-1}}\right)\left(2^{2^{k-1}}\right)+1=2^{2^{k}}+1$ there is a subsequence of the length $\left(2^{2^{k-1}}+1\right)$ monotone in the first coordinate. By induction this subsequence contains a 3-element subsequence monotone in all coordinates except the first one. But the whole subsequence is monotone in the first coordinate. So we have 3-element subsequence monotone in each coordinate. Hence by the previous lemma there are two $k$-gons such that one of them does not see the other one.

If $k$ is even we have a better estimate, as it is necessary to use only $k / 2$ coordinates. Hence we can get $f(2 k) \leq 2^{2^{k}}$. The same proof can be used to prove the theorem for polygons of different sizes with parallel edges. 


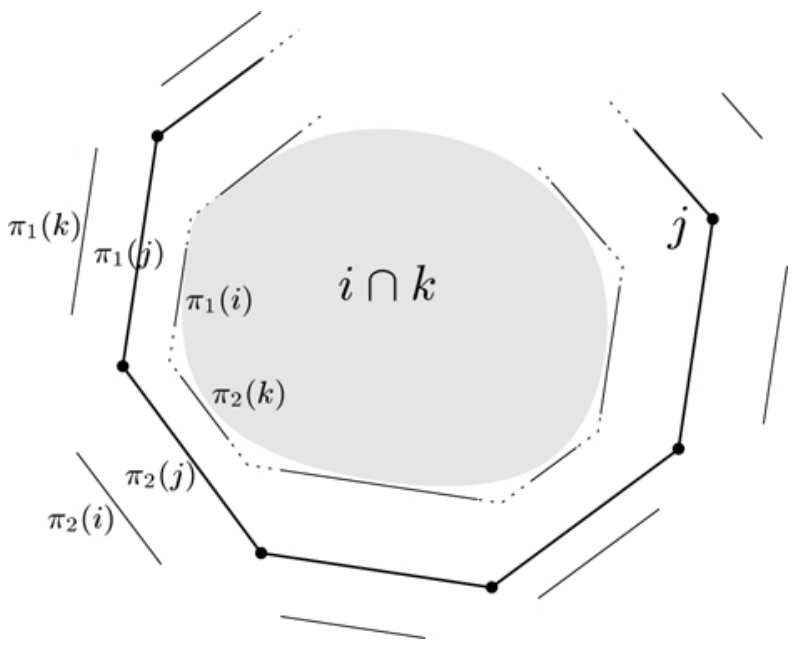

Fig. 4. Sequence of three polygons $i<j<k$ monotone in each coordinate

Conjecture 1 For every natural $k>4$, we conjecture $f(k) \leq f(k+2)$.

Conjecture 2 For every natural $k>4$, we conjecture $f(k) \leq f(2 k)$.

Conjecture 3 For every even $k$ it is possible to draw up an algorithm, which tells the maximum size of a configuration of $k$-gons, if we additionally require that the intersection of every pair of $k$-gons is a $k$-gon again.

The algorithm is similar to the one used in [FHW] for enumerating the maximum size of configuration of squares. If the $k$-gons lying in between $i$ and $j$ cover all $k$ vertices of the intersection $i \cap j$, then $i$ cannot see $j$. Thus all $k$-gons in the configuration see the others if and only if the following holds for every pair $i<j$ :

$$
\left|\bigcup_{i<m<j} \bigcap_{1 \leq l \leq k} P_{l}(m)\right|<k,
$$

where $P_{l}(m)$ is the set of vertices of the intersection $i \cap j$, which can be covered by $m$ in the direction of the $\pi_{l}$-coordinate.

\section{Triangles}

\section{Claim 6}

$$
f(3) \geq 14 \text {. }
$$

Proof. An example of configuration of fourteen triangles, which see each other is given in Figure 6. The orderings on the $\pi, \rho$ and $\sigma$ coordinates are given in the following table. 


\begin{tabular}{|c|cccccccccccccc|}
\hline$\pi$ & 5 & 4 & 10 & 8 & 7 & 2 & 6 & 1 & 3 & 14 & 13 & 12 & 11 & 9 \\
$\rho$ & 9 & 4 & 13 & 11 & 14 & 12 & 7 & 3 & 1 & 6 & 2 & 10 & 8 & 5 \\
$\sigma$ & 12 & 14 & 9 & 11 & 5 & 1 & 13 & 8 & 10 & 2 & 3 & 6 & 7 & 4 \\
\hline
\end{tabular}

We have developed a functional implementation of an algorithm which enumerates the upper bound for $f(3)$. It is based on the following three conditions the orderings on the three coordinates $\pi, \rho$ a $\sigma$ must satisfy, otherwise there is a pair of triangles not seeing each other.

Lemma 7 Every configuration of k-gons, has the following two properties:

1 There is no pair of $k$-gons $i \neq j$ such that

$$
\forall n=1, . ., k: \pi_{n}(i)<\pi_{n}(j),
$$

2 there is no triple $i<j<l$ such that

$$
\forall n=1, . ., k:\left(\left(\pi_{n}(i)<\pi_{n}(j)<\pi_{n}(l)\right) \vee\left(\pi_{n}(i)>\pi_{n}(j)>\pi_{n}(l)\right)\right.
$$

Remark: For even $k$ we use only $\pi_{1}, \ldots, \pi_{k / 2^{-}}$axis to describe the configuration; configuration satisfies 1 automatically.

First property follows from the fact that we have equal $k$-gons and therefore no $k$-gon can be smaller that any other in every coordinate. The second property is only a re-formulation of Lemma 5 , which says that there cannot be a subsequence monotone in all of the three coordinates, otherwise there is a pair of triangles not seeing each other.

Lemma 8 Every configuration of triangles has the following property:

3 If there is a subsequence monotone in the two coordinates, then the middle triangle must have the highest order in the third one.

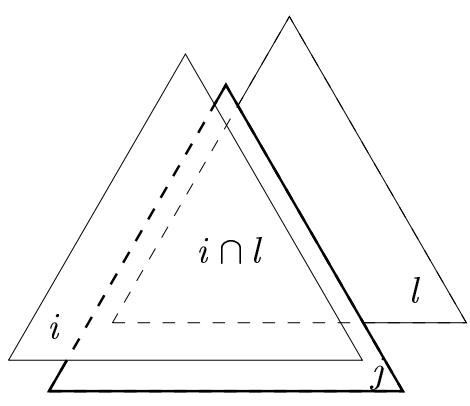

(a)

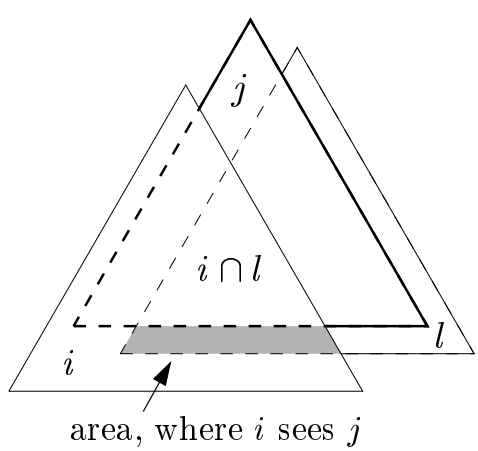

(b)

Fig. 5. Illustration to the property $\mathbf{3}$. 


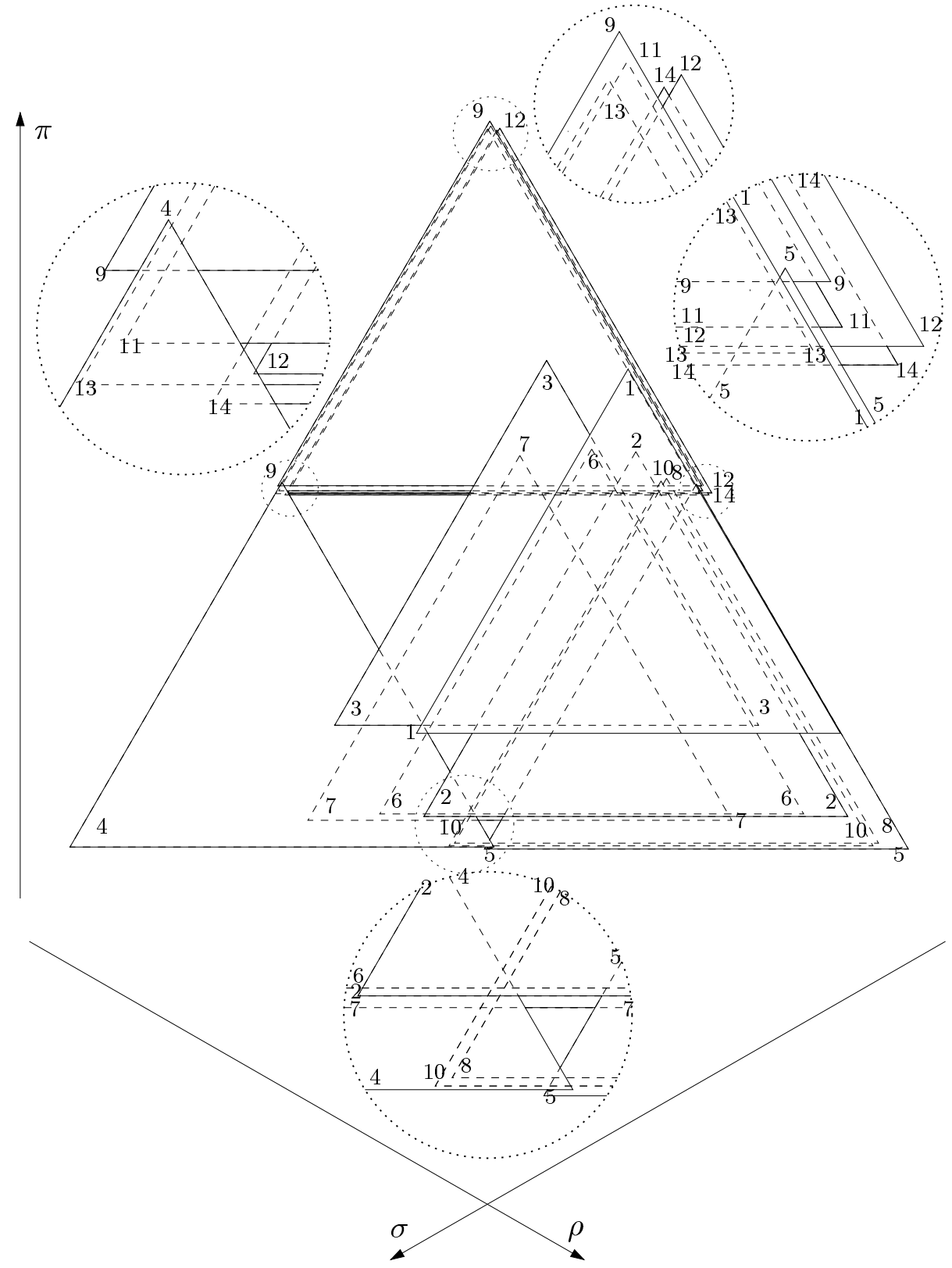

Fig. 6. Fourteen triangles seeing each other. 
Thus there is no triple $i<j<l$ such that

$$
\begin{gathered}
((\pi(i)<\pi(j)<\pi(l)) \vee(\pi(i)>\pi(j)>\pi(l))) \\
\wedge((\rho(i)<\rho(j)<\rho(l)) \vee(\rho(i)>\rho(j)>\rho(l))) \\
\wedge((\sigma(j)<\sigma(i)<\sigma(l)) \vee(\sigma(j)<\sigma(l)<\sigma(i))) .
\end{gathered}
$$

Proof. See Figure 5. We have three triangles $i<j<l$. In both figures is $(\rho(i)<\rho(j)<\rho(l))$ and $(\sigma(l)<\sigma(j)<\sigma(i))$ - a subsequence monotone in the two coordinates. In Figure $5(\mathrm{a})$ is $(\pi(j)<\pi(i)<\pi(l))$ and $j$ covers the intersection $i \cap j$ entirely, but in Figure $5(\mathrm{~b})$, where $(\pi(i)<\pi(l)<\pi(j))$, there is an area, where $i$ can see $j$.

We have stated two conjectures in connection with triangles.

Conjecture 4 Configuration is realizable (with no regard to the visibility) if and only if the orderings satisfy the property $\mathbf{1 .}$

Conjecture $\mathbf{5}$ It is possible to construct a configuration of triangles seeing each other if and only if the three orderings satisfy the properties $\mathbf{1}, \mathbf{2}$ and $\mathbf{3}$.

The result we obtain from the computer is a triple of sequences satisfying the necessary conditions - the upper bound. We have to give an example of the corresponding number of triangles each seeing all the others. We would not need that once we have proved Conjecture 5 .

After searching through approximately one third of all possibilities, we have the lower bound $f(3) \leq 14$ and we conjecture that it is the final result.

\section{Open problems}

1 To prove or refute the conjectures mentioned in the previous.

2 We want to draw all combinatorially distinct configurations of triangles into a triangle net. How fine this net has to be?

3 What can be said about the function $f(k) / k$ ? Does the limit $\lim _{k \rightarrow \infty} \frac{f(k)}{k}$ exist?

\section{References}

AGW. H. Alt, M. Godau, S. Whitesides: Universal 3-Dimensional Visibility Representations for Graphs Proc. Graph Drawing '95, Passau, 1995. Lecture Notes in Computer Science LNCS \#1027, Springer-Verlag, 1996, pp. 8-19

FHW. S. P. Fekete, M. E. Houle, S. Whitesides: New Results on a Visibility Representation of Graphs in 3D Proc. Graph Drawing '95, Passau, 1995. Lecture Notes in Computer Science LNCS \#1027, Springer-Verlag, 1996, pp. 234-241 